ARTICLE

https://doi.org/10.1038/s41467-019-13979-5

\title{
The nuclear pore complex prevents sister chromatid recombination during replicative senescence
}

\author{
Paula Aguilera', Jenna Whalen ${ }^{2,3}$, Christopher Minguet ${ }^{1,3}$, Dmitri Churikov ${ }^{1}$, Catherine Freudenreich (iD ${ }^{2}$, \\ Marie-Noëlle Simon ${ }^{1 \star} \&$ Vincent Géli ${ }^{1 \star}$
}

The Nuclear Pore Complex (NPC) has emerged as an important hub for processing various types of DNA damage. Here, we uncover that fusing a DNA binding domain to the NPC basket protein Nup1 reduces telomere relocalization to nuclear pores early after telomerase inactivation. This Nup1 modification also impairs the relocalization to the NPC of expanded CAG/CTG triplet repeats. Strikingly, telomerase negative cells bypass senescence when expressing this Nup1 modification by maintaining a minimal telomere length compatible with proliferation through rampant unequal exchanges between sister chromatids. We further report that a Nup1 mutant lacking 36 C-terminal residues recapitulates the phenotypes of the Nup1-LexA fusion indicating a direct role of Nup1 in the relocation of stalled forks to NPCs and restriction of error-prone recombination between repeated sequences. Our results reveal a new mode of telomere maintenance that could shed light on how $20 \%$ of cancer cells are maintained without telomerase or ALT.

\footnotetext{
${ }^{1}$ Marseille Cancer Research Center (CRCM), U1068 Inserm, UMR7258 CNRS, Aix Marseille University, Institut Paoli-Calmettes Equipe labellisée Ligue, 27 bd Lei Roure, Marseille, France. ${ }^{2}$ Department of Biology, Tufts University, 200 Boston Ave, Medford, MA 02155, USA. ${ }^{3}$ These authors contributed equally: Jenna Whalen, Christopher Minguet. *email: marie-noelle.simon@inserm.fr; vincent.geli@inserm.fr
} 
T elomeres are nucleoprotein structures that protect the ends of linear eukaryotic chromosomes against degradation, end-to-end fusions and homologous recombination (HR). They consist of G-rich repetitive DNA sequences with a terminal $3^{\prime}$ single-strand overhang ${ }^{1}$. Capping of telomeres is necessary to avoid their recognition as double-strand breaks (DSBs) and resulting genome instability ${ }^{2}$. In Saccharomyces cerevisiae, telomeres consist of an array of about 300-bp of $\mathrm{TG}_{1-3}$ repeats and a 12-14-nucleotide-long $3^{\prime}$ single-strand overhang. The Rap1 protein wraps the double-stranded telomeric DNA to inhibit end fusion $^{3}$ and recruits Rif1 and Rif2 that limit $\mathrm{HR}^{4-6}$. The $3^{\prime}$ overhang is bound by Cdc13, a subunit of the CST (Cdc13/Stn1/ Ten1) complex ${ }^{7}$. The specific sequence and chromatin properties of telomeres create a challenge for the DNA replication machinery that requires accessory factors at telomeres in both yeast and mammals ${ }^{8,9}$.

Telomere length is carefully regulated, and is maintained by the reverse transcriptase telomerase to bypass the end replication problem ${ }^{10}$. As in metazoans, inactivation of telomerase in yeast leads to progressive shortening of telomeres until they reach a critical length that impairs their capping function. When telomeres become critically short, unprotected telomeres elicit a DNA damage response (DDR), recruit Mec1 ${ }^{\mathrm{ATR}}$ and activate a permanent $\mathrm{G} 2 / \mathrm{M}$ arrest ${ }^{11,12}$ leading to replicative senescence. However, telomerase is not only required to replenish chromosome ends upon their gradual erosion but also essential to counteract replication-induced damage at telomeres ${ }^{13-15}$. Therefore, in the absence of telomerase, telomere replication becomes imminently dependent on HR factors. As a consequence, the deletion of RAD52 or RAD51 dramatically accelerates senescence ${ }^{16}$, although how HR resolves replication stress remains poorly understood.

The progressive loss of the growth capacity observed upon telomerase inactivation thus stems from two distinct types of damage, i.e., stochastic replication fork stallings that induce transient cell-cycle arrests soon after telomerase inactivation ${ }^{17,18}$ and gradual erosion of the telomeres from their termini that eventually induces permanent cell-cycle arrests ${ }^{12}$. A small fraction of the permanently arrested cells can bypass senescence and resume divisions by regenerating functional telomeres through recombination ${ }^{16}$. Two types of survivors are described in budding yeast. Type I survivors amplify $\mathrm{Y}^{\prime}$ subtelomeric sequences separated by short interstitial $\mathrm{TG}_{1-3}$ repeats while maintaining very short telomere ends. Type II survivors have very long $\mathrm{TG}_{1-3}$ tracts, heterogeneous in length ${ }^{16}$ that resemble telomeres in the cancer cells that maintain their telomeres via alternative lengthening of telomeres (ALT). Both types of repair use break-induced replication (BIR) dependent on Rad52, Pol32 and Pif1 16,19,20. Type I recombination requires in addition Rad51, Rad54 and Rad57, while Type II recombination depends on Rad59, the MRX (Mre11/Rad50/Xrs2) complex, Sgs1 and Sae2 21-25.

DNA damage can be repaired by multiple and sometimes redundant mechanisms with variable capacities to preserve genetic information and genome stability. Repair of DNA damage appears to be spatially segregated within the nucleus ${ }^{26,27}$. In budding yeast, persistent DSBs, usually those that lack a homologous sequence for repair, are often shifted to the vicinity of the nuclear envelope $(\mathrm{NE})^{28,29}$. One key structure at the $\mathrm{NE}$ is the nuclear pore complex (NPC) that forms an aqueous channel embedded in the nuclear membrane. NPCs consist of a central ring structure in the plane of the NE that extends as cytoplasmic filaments on one side and the nuclear basket on the other side ${ }^{30}$. Integrity of the NPC is required for proper response to DNA damage, as mutating NPC components confers sensitivity to DNA-damaging agents ${ }^{31-33}$. Similarly to persistent DSBs, highly eroded and poly-SUMOylated telomeres shift to the nuclear pores $^{34,35}$.

We showed that relocalization of eroded telomeres to the nuclear pores favors type II recombination ${ }^{35}$. This observation was in line with the view that NPC relocalization promotes noncanonical repair of congested DNA structures using error-prone last chance mechanisms such as BIR or microhomology-mediated end-joining ${ }^{36}$. Nevertheless, the role of the nuclear pore might extend to other types of telomere damage as some damaged telomeres are detected at the NPCs early after telomerase inactivation and might represent telomeres under replication stress ${ }^{34}$. Indeed, the replication forks that confront a barrier formed by expanded CAG/CTG triplet repeats transiently shift to the NPCs, a mechanism that prevents repeat fragility and instability and is thus hypothesized to promote fork restart ${ }^{37}$.

In this study, we uncover that modification of the NPC basket protein Nup1 impairs the relocalization to NPCs of the telomeres under replication stress in telomerase-negative as well as expanded CAG/CTG triplet repeats in telomerase-positive cells. Strikingly, in telomerase-negative cells expressing modified Nup1, telomeres are maintained for generations at a short but functional length. This telomere maintenance mechanism that relies on nonconservative exchanges between sister chromatids is sufficient to avoid the proliferative decline and telomere shortening-driven crisis normally observed in telomerase-negative cells. Collectively, our results unveil an unsuspected role of the NPC in suppressing sister-chromatid recombination (SCR) at telomeres and reveal a new mechanism of telomere maintenance.

\section{Results}

Fusion of LexA to Nup1 prevents senescence in the absence of telomerase. In order to address the role of the NPC in controlling telomere recombination during replicative senescence ${ }^{35}$, we constructed a strain in which Nup1 was C-terminally tagged at its native locus with the DNA binding protein LexA (Fig. 1a). Our initial idea was to develop an assay to tether genomic regions tagged with LexA-binding sites to the NPC in telomerase-negative cells. In contrast to the deletion of NUP1, the Nup1-LexA fusion was viable and did not impair cell proliferation (Supplementary Fig. 1a, b). It also showed no additive effects with the absence of the mitotic exit regulator Bub2 (Supplementary Fig. 1b) that aggravates the growth defect of NUP1-deficient cells ${ }^{38}$. Importantly, the SUMO-protease Ulp1 was properly localized to the nuclear rim in nup1-LexA cells (Supplementary Fig. 1c). Furthermore, quantitative assessment of the nuclear export of poly (A)+-RNA and of the nucleocytoplasmic transport of proteins did not reveal any gross alterations in nup1-LexA-expressing cells as compared to WT cells (Supplementary Fig. 1d-f). Thus, in all these respects, the nup1-LexA strain did not have the defects of the NUP1-deficient strain.

The haploid nup1-LexA strain was crossed with a telomerasenegative est2 $\Delta$ strain maintained alive with an EST2-expressing plasmid. Haploid est $2 \Delta$ spore clones were isolated after micromanipulation of the spores on plates and then propagated in liquid cultures for about 120 population doublings (PDs) via serial dilutions every $24 \mathrm{~h}$. The growth capacity of the clonal cell populations was assessed by measurement of the cell density every day. As expected, proliferation of the control est $2 \Delta$ cells declined progressively until the culture entered telomere-erosiondriven crisis after about 70 PDs before formation of the survivors (Fig. 1b). In striking contrast, est2A nup1-LexA clones greatly attenuated the loss of proliferative capacity and essentially bypassed senescence (Fig. 1b). Importantly, these results were obtained in est2A nup1-LexA cells lacking any LexA-binding sites. 
$\mathbf{a}$
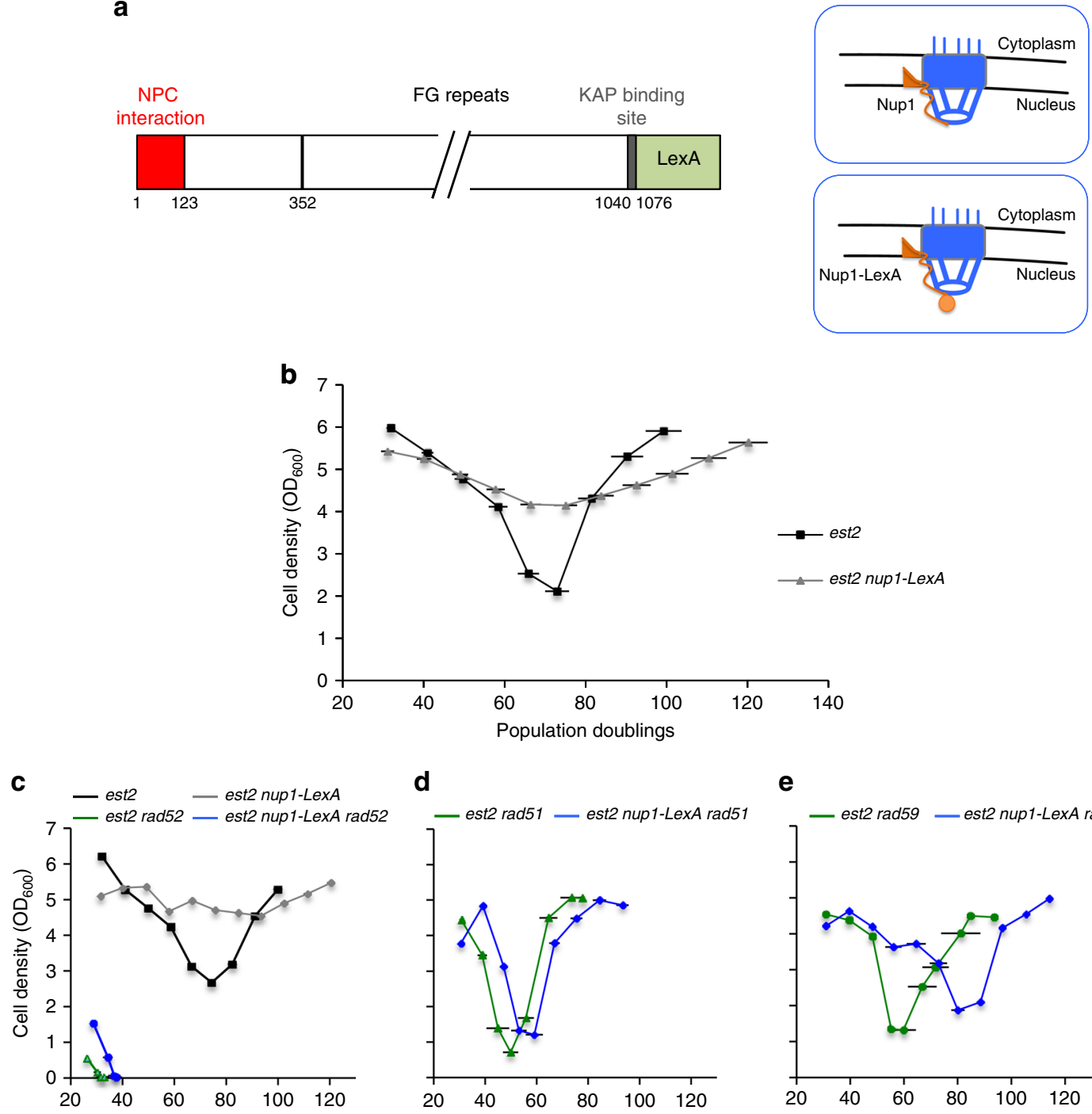

d

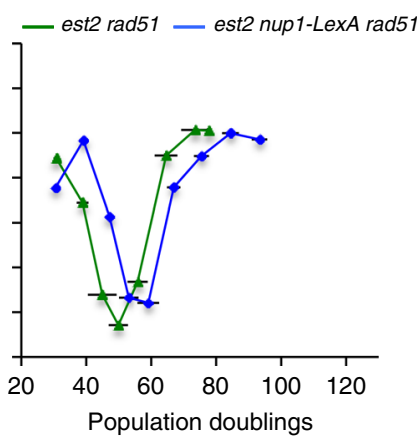

e

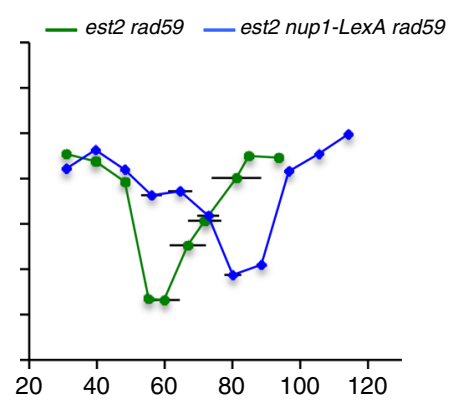

Fig. 1 Attenuation of senescence in est2 $\Delta$ nup1-LexA cells. a Schematic structure of the nuclear basket protein Nup1 fused to LexA. b Mean senescence profiles of the est2 $(n=22)$ and est2 nup1-LexA $(n=28)$ clones analyzed in the course of this study. Each clone was isolated by sporulation of a heterozygous diploid strain. Each spore colony was then propagated in liquid culture through daily serial dilutions. $\mathrm{OD}_{600}$ was measured every day to estimate the cell density reached in $24 \mathrm{~h}$. PD numbers were estimated from the initial spores. c Mean senescence profiles of est2 $\Delta$ and est2 $\Delta$ nup 1 -LexA clones in the absence of RAD52 ( $n=4$ and 8, respectively). Control est2 $(n=7)$ and est2 $\triangle$ nup1-LexA $(n=5)$ curves are from the clones used in this specific experiment. d Mean senescence profiles of est2 $\Delta$ and est2 $\Delta$ nup1-LexA clones in the absence of RAD51 ( $n=4$ and 7, respectively). e Mean senescence profiles of est $2 \Delta$ and est2 2 nup1-LexA clones in the absence of RAD59 ( $n=4$ and 6 , respectively). Error bars indicate SD.

To determine whether the nup1-LexA allele confers a defect in DNA damage checkpoint activation, we measured the sensitivity of telomerase-positive nup1-LexA cells to hydroxyurea (HU), methyl methanesulfonate (MMS) and UV irradiation. We detected neither increased sensitivity nor resistance of nup1LexA cells to HU, MMS or UV irradiation (Supplementary Fig. 1g). Accordingly, untreated nup1-LexA cells did not display auto-phosphorylation of Rad53 as observed in the presence of MMS (Supplementary Fig. 1h).

Taken together, these data indicate that although the Nup1LexA fusion protein appeared largely functional with respect to Nup1 canonical functions, its expression suppressed the senescence phenotype of the telomerase-negative cells.

Senescence bypass by the est2A nup1-LexA cells requires HR factors. We sought to determine whether the ability of nup1-LexA cells to attenuate senescence requires HR components. We first deleted RAD52 in est2 $\triangle$ nup1-LexA cells and re-examined their growth. RAD52 deletion induced a premature and definitive growth arrest in nup1-LexA est $2 \Delta$ cells as it did in the control cells (Fig. 1c). In contrast, rad52 $\Delta$ did not affect growth of nup1-LexA cells expressing telomerase (Supplementary Fig. 1i). We next tested the role of Rad51 and Rad59 that define two distinct pathways of Rad52-dependent $\mathrm{HR}^{39}$. We observed that the role of HR in maintaining growth of est $2 \Delta$ nup1-LexA cells relied on Rad51 as senescence was no longer attenuated by nup1-LexA in its absence (Fig. 1d). The rescue depended on Rad59 as well but to a lesser extent, as senescence was re-established but was delayed (Fig. 1e). Taken together, these results suggest that steady proliferation of the est2 nup1-LexA cells was dependent on HR.

Nup1-LexA impairs the localization of the early telomere damage. We showed that accumulation of single-strand DNA at a single telomere is sufficient to produce a fluorescent focus in cells 
a
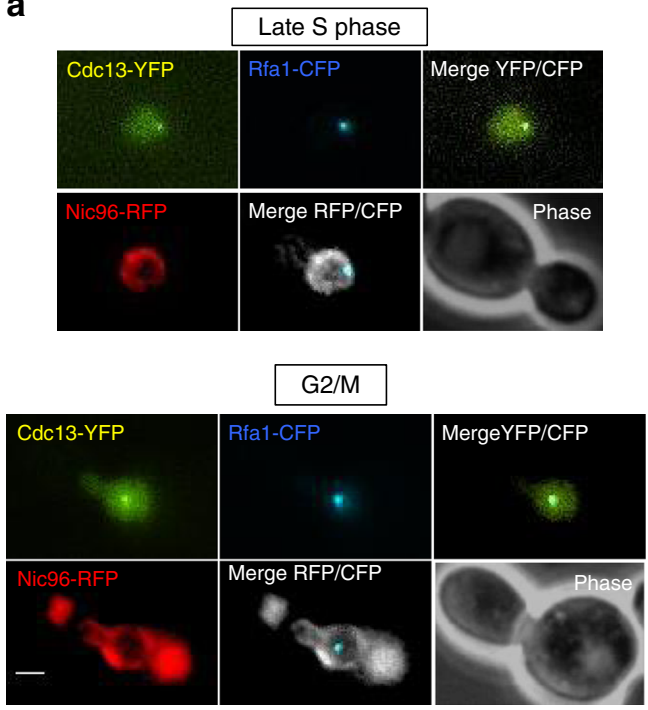

b
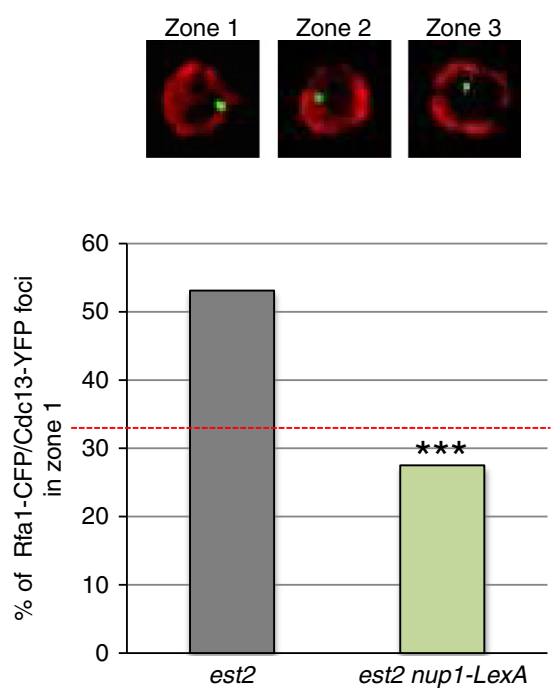

Fig. 2 Nup1-LexA alters the localization of damaged telomeres during senescence. a Damaged telomeres were detected as foci containing both Cdc13YFP and Rfa1-CFP and localized relative to Nic96-RFP that marks the nuclear periphery. Representative images of late S (upper panel) and G2/M (lower panel) cells are shown. Scale bar: $2 \mu \mathrm{m}$. b The localization of the Cdc13-YFP/Rfa1-CFP foci in the three zones of equal area of the nucleus marked by Nic96RFP (upper panel) was scored in late $\mathrm{S}$ and G2/M cells. Cells were imaged after one restreak from spore colonies (about 35 PDs from the initial spores). The percentages of zone 1 foci are from analysis of two independently isolated est $2 \Delta$ ( $n=96$ cells) and est $2 \Delta$ nup 1 -LexA ( $n=120$ cells) clones. The analysis of the repartition of Rfa1/Cdc13 foci between the three equal zones of the nucleus is shown for individual clones in Supplementary Fig. 2a. Statistical differences were determined by a Fisher's exact test $\left({ }^{\star \star \star} p=0.0001\right)$.

expressing Cdc13-YFP 34 . Cdc13 foci are detected as soon as 20 PDs after telomerase inactivation and localize to the nuclear periphery. We inferred that these early Cdc13-YFP foci that are also enriched in Rfal may represent telomeric stalled replication forks ${ }^{40}$. To determine whether expression of Nup1-LexA interferes with the localization of early telomere damage, we performed a zoning assay in est $2 \Delta$ and est $2 \Delta$ nup1-LexA cells. In this assay, the nucleus is divided into three zones of equal volume, and the position of a given focus relative to the nuclear periphery, here defined by Nic96-RFP, is scored ${ }^{41}$. To visualize telomeric stalled forks, we analyzed the colocalization of Rfa1-CFP foci with Cdc13-YFP foci from two independently isolated est $2 \Delta$ and est $2 \Delta$ nup1-LexA clones after one restreak on yeast extract peptone dextrose (YPD) plates (about 30 PDs after telomerase inactivation) (Fig. 2a). The localization of the Cdc13-YFP/Rfa1-CFP foci was then scored in S/G2-M cells into one of three nuclear zones (Supplementary Fig. 2). Remarkably, the peripheral enrichment of the Cdc13-YFP/Rfa1-CFP foci observed in the est2 $\Delta$ cells was totally abolished in the nup1-LexA-expressing cells (Fig. 2b and Supplementary Fig. 2).

Nup1-LexA impairs triplet repeat stability and relocalization to NPC. It was proposed that replication fork repair can occur either in the interior of the nucleus or at the nuclear periphery depending on the state of the arrested fork ${ }^{42}$. HU-stalled forks or forks that encounter a nick do not localize to the nuclear pore 29,43 while forks that encounter a naturally occurring barrier such as expanded CAG trinucleotide repeats $\mathrm{do}^{37}$. We therefore tested whether the localization of CAG sequences containing 130 repeats tagged with a lacO array was altered in the presence of Nup1-LexA in telomerase-positive cells. To this purpose, we scored in S-phase the position of GFP-LacI foci relative to the nuclear periphery ${ }^{37}$ (Fig. 3a). Samples were taken after each growth step to confirm that the tract length was not contracted in the cells used to start the assay (see Methods). Strikingly, the peripheral enrichment of the repeat locus observed in the WT strain was lost in the nup1-LexA-expressing cells (Fig. 3b) suggesting that the fusion protein induced a general defect in the relocalization to the NPC of replication stress-induced damage. To further investigate the consequences of nup1-LexA expression in the processing of replication-induced damage, we monitored instability (expansions and contractions) (Fig. 3c) and fragility (breakage) (Supplementary Fig. 3a) of long CAG/CTG repeat tracts using established assays ${ }^{37}$. Expression of Nup1-LexA did not exacerbate the fragility of either CAG-70 or CAG-155 expanded repeats (Supplementary Fig. 3b). In contrast, it significantly affected the stability of the repeats and favored contractions at the expense of expansions (Fig. 3c). Notably the contractions were strongly reduced by inactivation of Rad52 (Fig. 3c), suggesting that nup1-LexA expression unleashes an HR pathway that is normally restricted when stalled forks at CAG repeats are relocalized to the NPC.

Telomeres are maintained at a minimal length in est2 $\Delta$ nup1LexA cells. To assess the state of telomeres during replicative senescence in telomerase-negative cells expressing Nup1-LexA, telomere length was analyzed by Southern blot at different time points of the senescence in several independently isolated clones. Figure 4 shows representative Southern blots of three of these clones. In all the clones analyzed, telomeres shortened as a function of the PDs in est2 nup1-LexA cells as they did in the control est $2 \Delta$ cells. As expected, the liquid cultures of est $2 \Delta$ cells after the senescence crisis were dominated by type II survivors because of their growth advantage over type I survivors (Fig. 4a, b). In striking contrast, est $2 \Delta$ nup1-LexA clones maintained short tracts of $\mathrm{TG}_{1-3}$ repeats for up to $130 \mathrm{PDs}$ (Fig. $4 \mathrm{~b}$ ). Out of the 25 independent clones analyzed during the course of this study, six maintained short telomere tracts without any signs of either $\mathrm{Y}^{\prime}$ or $\mathrm{TG}_{1-3}$ repeat amplification (Fig. $4 \mathrm{~b}$, clone \#6). Nine showed some bands corresponding to type II recombination at the late time points while still maintaining short terminal $\mathrm{TG}_{1-3}$ repeats at some telomeres (Fig. 4b, clone \#A11). The remaining ten clones displayed $\mathrm{Y}^{\prime}$ amplification reminiscent of type I recombination at late time points (Fig. 4b, clone \#A45). These results are consistent 
a

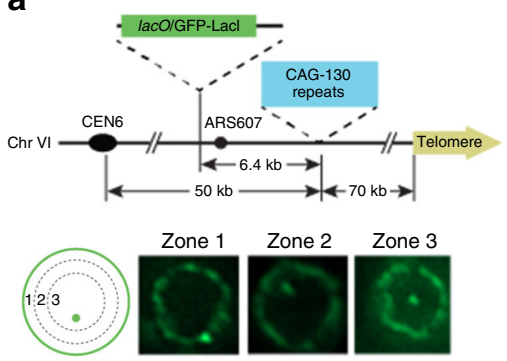

b CAG-130, S-phase

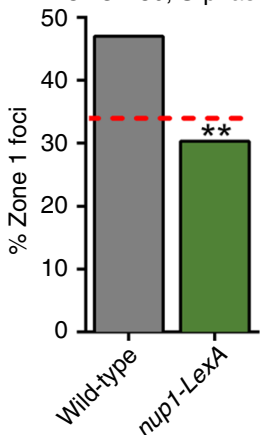

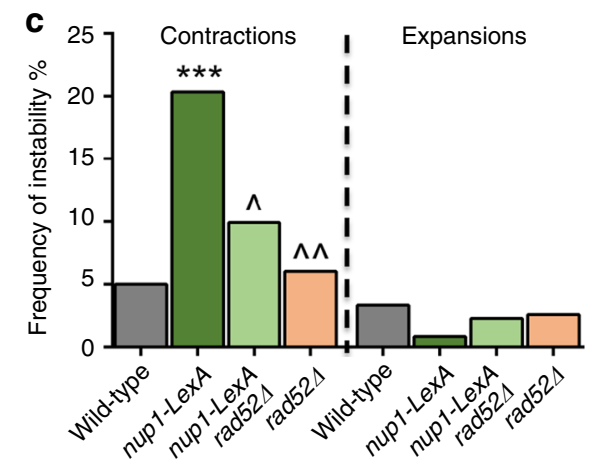

\begin{tabular}{|c|c|c|c|c|c|}
\hline & \multicolumn{4}{|c|}{ CAG-70 } & \multirow{2}{*}{$\begin{array}{c}\text { Total \# of } \\
\text { colonies } \\
\text { tested }\end{array}$} \\
\hline & Contractions & \multicolumn{2}{|c|}{ Expansions } \\
\cline { 1 - 5 } & $\#$ & $\%$ & $\#$ & $\%$ & 279 \\
\hline Wild-type & 14 & 5.0 & 9 & 3.2 & 279 \\
\hline nup1-LexA & 24 & 20 & 1 & 0.85 & 118 \\
\hline $\begin{array}{c}\text { nup1-LexA } \\
\text { rad524 }\end{array}$ & 13 & 9.9 & 3 & 2.3 & 131 \\
\hline rad524 & 7 & 6.0 & 3 & 2.6 & 116 \\
\hline
\end{tabular}

Fig. 3 Nup1-LexA affects localization and stability of CAG trinucleotide repeats. a Yeast chromosome VI containing 130 integrated CAG repeats and a lacO array that binds the Lacl-GFP protein. S-phase cells were imaged, and the location of the foci was scored into one of three zones of equal area, using the GFP-Nup49 signal to mark the nuclear periphery. b Percent of zone 1 foci for CAG-130 S-phase cells (283 cells for wild-type, 142 cells for nup1-LexA). Statistical differences were determined by a Fisher's exact test ( $\left.{ }^{\star *} p=0.0012\right)$. c Frequency of contractions and expansions for CAG-70 repeats in wild-type and nup1-LexA cells. The frequency was determined by analysis of PCR amplicon length on a highresolution fragment analyzer gel system, using PCR primers that flank the CAG-70 tract located on a YAC (see Fig. S6A); $\left.{ }^{(\star \star}\right) p=0.0001$ compared with wild-type by Fisher's exact test; $\left({ }^{\wedge}\right) p=0.0313 ;\left(^{\wedge}\right) p=0.0017$ compared with nup1-LexA by Fisher's exact test.

with a scenario where telomeres do not reach the critical length that induces permanent checkpoint activation but instead are maintained at a minimal length compatible with cell proliferation, and in so doing either prevent or postpone the types of recombination that lead to survivor formation.

Telomeres are maintained by recombination with the sister chromatid. To further investigate the type of repair occurring at telomeres in est2A nup1-LexA cells, we used telomere PCR to clone and sequence a subset of one telomere (TelVI-R) from clonal populations before cells enter the nadir of senescence using a specific TELVI-R probe that hybridizes just upstream the $\mathrm{TG}_{1-3}$

repeats (Supplementary Fig. 4a, Day 5). Each sequence was compared with the reference sequence obtained from the same clone at the earliest time point (Day 1). The telomerase of $S$. cerevisiae has the peculiarity to add $\mathrm{TG}_{1-3}$ repeats of variable length ${ }^{44}$, and consequently the sequence of each individual telomere is unique and stable over generations provided that its distal region is not elongated by telomerase or modified through recombination with another telomere. Alignment of multiple copies of TelVI- $R$ from est $2 \Delta$ cells showed that, as expected, a large majority (94\%) of the sequences matched perfectly with the reference sequence (Fig. 5a). In line with previous publication ${ }^{22}$, $6 \%$ of the telomeres showed sequence divergence in the distal region that might stem from rare recombination events or from technical reasons related to PCR amplification and subcloning 45 . In contrast, more than a quarter of TelVI-R sequences from est $2 \Delta$ nup1-LexA could not be perfectly aligned with the reference sequence over their total length (results from two independent clones are shown in Fig. 5a and Supplementary Fig. 4a-c).

Careful analysis of each misaligned part of the sequences showed that they can be all matched to the reference sequence of TELVI-R provided that either a gap or an insertion was introduced (Fig. 5b, clones al and e1, respectively). Because each telomere sequence is unique, this finding ruled out a possibility that seemingly divergent telomere sequences were a result of recombination with another telomere. Instead, gapped alignment is more consistent with SCR events. Notably, deletions always occurred within repeated motifs at the breakpoint (Fig. 5b, clone al, repeated motif indicated by blue sequence). The occurrence of insertions was evident by starting the alignment upstream from the point of divergence (Fig. 5b, clone e1) indicating that duplication occurred during repair. Equal SCR normally generates repair products without change in DNA sequence. At telomeres, however, the nature of the repeats provides conserved motifs at several positions where a D-loop can be initiated (Fig. 5c). Shifted annealing with an upstream or downstream conserved motif would therefore produce a shorter or elongated telomere, respectively (Fig. 5c). In vivo, a 5 - to 8-bp homologous tail at the $3^{\prime}$ end appears to be sufficient to assure recombination even in the presence of single-base-pair mismatches if the heteroduplex can be extended ${ }^{46}$. These sequencing results unveil a new mode of telomere maintenance (distinct from type I and II recombination), which operates early after telomerase inactivation in cells expressing nup 1-LexA but is likely largely repressed in est2 $2 \Delta U P 1+$ cells.

Interestingly, although the helicase/ubiquitin ligase Rad5 acts in parallel to HR to delay senescence ${ }^{47}, R A D 5$ deletion did not affect the continuous growth of est2 $\Delta$ nup1-LexA cells (Supplementary Fig. 4d). This result suggests that Nup1-LexA overrides the requirement for the Rad5-dependent template switch branch of the DNA damage tolerance (DDT) pathway. In contrast, the helicase Srs2 played a key role in the continuous growth of est2A nup1-LexA cells, whereas its deletion only slightly affected the rate of senescence in est2 $\Delta$ control cells (Supplementary Fig. $4 \mathrm{e}$ and ref. ${ }^{48}$ ). This result is in line with the role of Srs2 in promoting replication fork restart via template exchange at a natural protein/DNA replication barrier ${ }^{49}$. Srs2 helicase activity has also been shown to be important for fork restart at an expanded CAG/CTG tract fork barrier ${ }^{50}$. Finally, we found that Pif1 was required for the steady growth of est2 $2 \Delta u p 1-$ LexA cells suggesting that BIR is involved in maintaining functional telomeres in the absence of telomerase (Supplementary Fig. 4f). From these data, we conclude that Nup1-LexA expression confers permissive conditions for telomere repair by SCR that is normally restricted in WT cells.

These data therefore raised the possibility that Nup1-LexA favors repair of telomeres by SCR by hindering their 


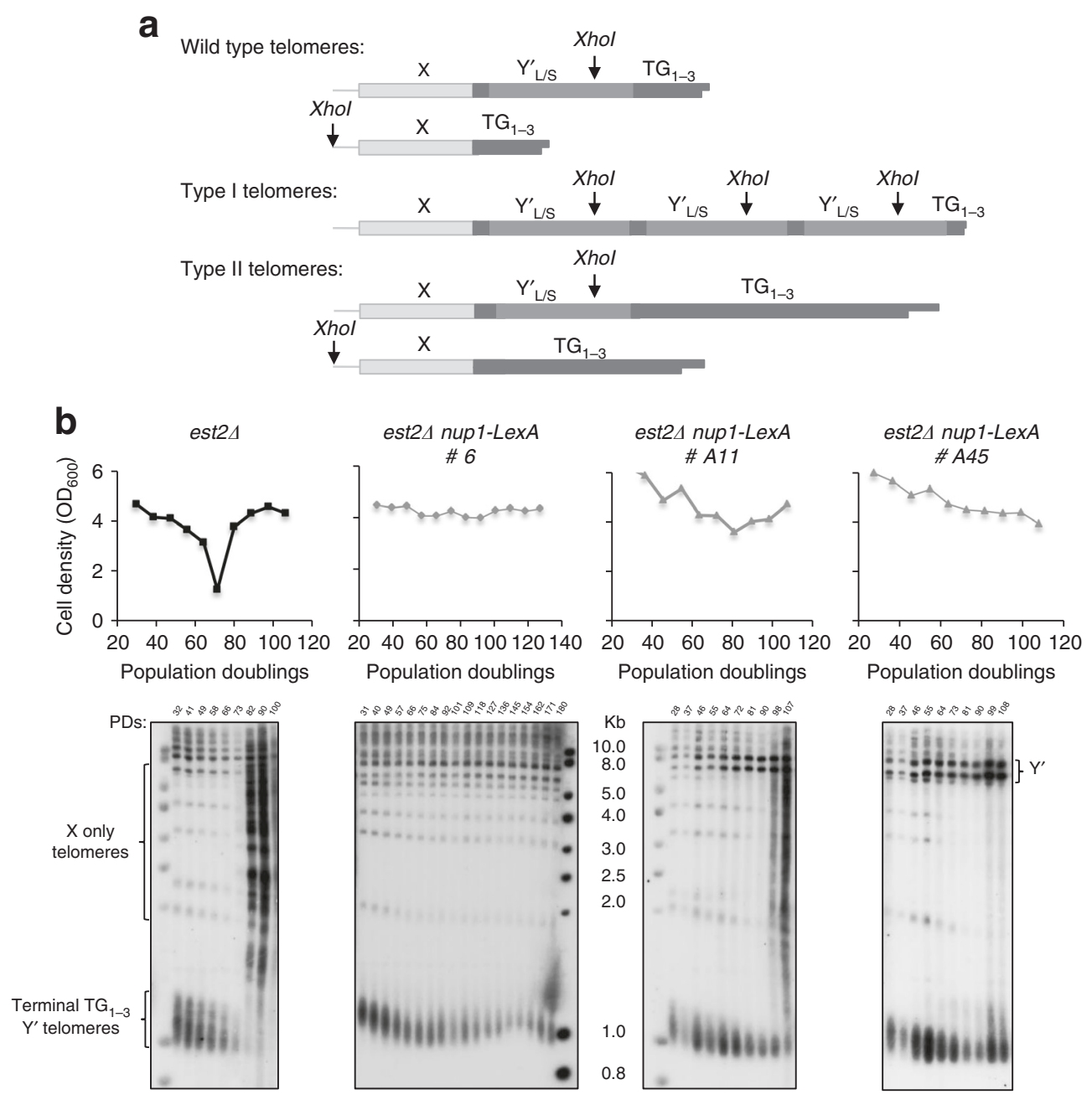

Fig. 4 Telomere length is maintained at a minimal length in nup1-LexA cells. a Schematic representation of wild-type, type I and type II telomeres. All telomeres contain one $X$ element in the subtelomeric region. In addition, about two-third of the telomeres contain from one to four long ( $L$ ) or short ( $\mathrm{S}$ ) subtelomeric sequences called $Y^{\prime}$ separated by short interstitial $\mathrm{TG}_{1-3}$ repeats, both being amplified in type I survivors. Positions of the Xhol sites are shown. $\mathbf{b}$ Senescence profiles of one est $2 \Delta$ clone (black) and three independent representative est2 $\Delta$ nup 1 -LexA clones (gray). Telomere length and survivor formation in the same replicative senescence experiment were monitored by $\mathrm{TG}_{1-3}$ probed Southern blots of Xhol-digested DNA.

relocalization to the NPC. To further address this possibility, we forced the localization of one specific telomere to the NPC in cells expressing Nup1-LexA. To this end, we introduced eight LexAbinding sites proximal to telomere VI-R in est2A nup1-LexA cells (Fig. $6 \mathrm{a}$ and ref. ${ }^{35}$ ). The supplementary Fig. $5 \mathrm{~b}$ shows the mean senescence curve of 11 independent est2 $\Delta$ nup1-LexA TelVI-R8LexAbs clones. As expected, the presence of a single telomere tethered to the NPC was not sufficient to significantly drive est $2 \Delta$ nup1-LexA cells to senescence crisis. We thus evaluated the frequency of TelVI-R recombination events in est2A nup1-LexA TelVI-R-8LexAbs cells by sequencing a subset of telomeres as described above. Analysis of two independent clones showed that the occurrence of rearrangements was decreased from $26.5-28 \%$ to only $9-16 \%$ at TelVI-R-8LexAbs (Fig. 6b, c and Supplementary Fig. 5c). Southern blot analysis of terminal restriction fragments containing TelVI-R revealed that tethering TelVI-R to the pore increased the frequency of type II recombination events (Supplementary Fig. 5d, e). Together these results suggest that tethering a single telomere to the pore decreases SCR and the peculiar telomere maintenance mode observed in nup1-LexA cells. Moreover, it restores the odds of type II recombination typical to telomeres in est $2 \Delta$ cells with unmodified Nup1.
Rad53 is required to sustain growth of est2A nup1-LexA cells. As mentioned above, stochastic transient cell cycle arrests occur in the heterogeneous population of senescing cells that are distinct from the terminal arrest induced by critically short telomeres ${ }^{18}$. Transient and terminal arrests are both dependent on checkpoint activation ${ }^{18}$. In particular, Rad53 was shown to have multiple roles at stalled replication forks including replisome stabilization and fork restart ${ }^{51,52}$. Rad53 was also proposed to release transcribed genes from the NPC when a replication fork encounters nuclear pore-gated transcripts ${ }^{53}$. We tested the role of the checkpoint effector kinase Rad53 in sustaining the growth of the est2 2 nup1LexA cells by evaluating the senescence profiles of est $2 \Delta$ nup1LexA clones carrying the checkpoint deficient rad53-K227A allele. Strikingly, inactivation of Rad53 completely abolished the effect of Nup1-LexA on senescence and telomere maintenance (Supplementary Fig. 6a). This shows that the activity of Rad53 is required for SCR at telomeres in est2 n nup1-LexA cells.

We also tested whether the SUMO pathway is involved in the unusual SCR occurring in the presence of Nup1-LexA. To this purpose, we introduced deletions of the two main E3 SUMOligase genes (SIZ1 and SIZ2) in est2A nup1-LexA cells and monitored the senescence profiles of independent clones. Deleting 
a

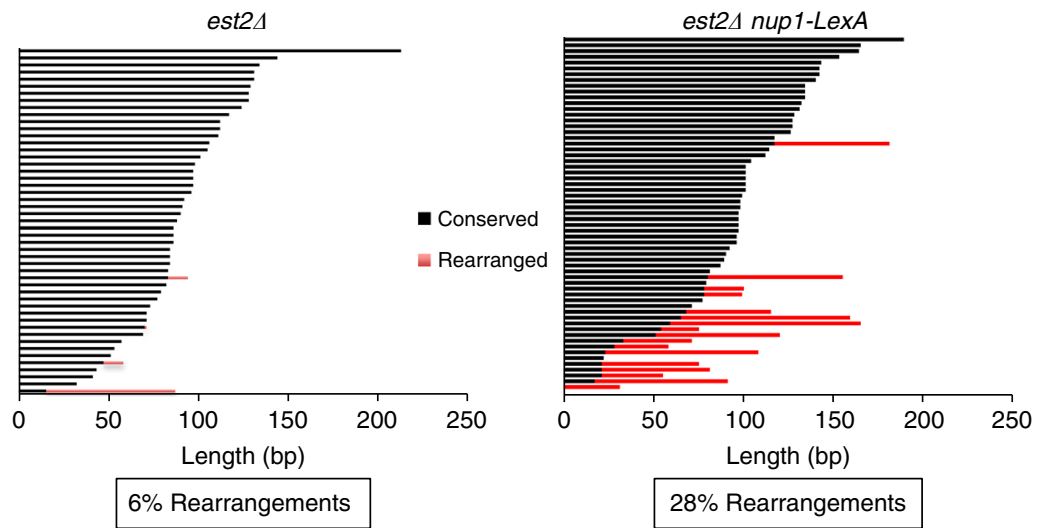

b

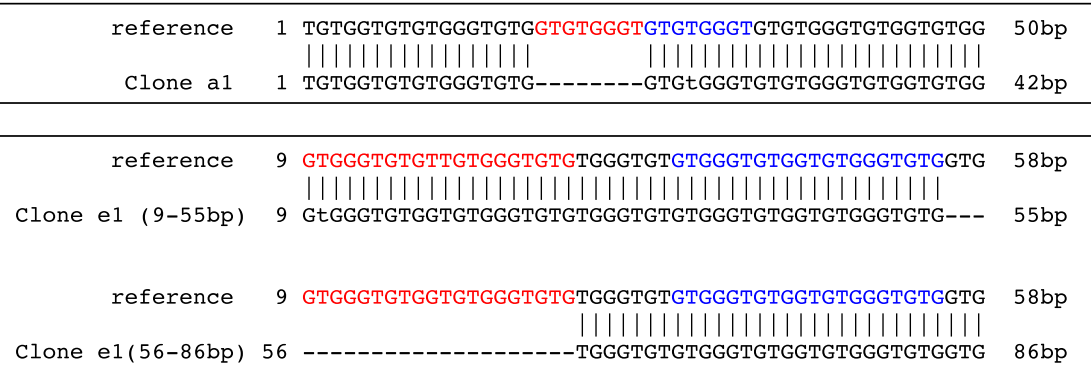

C

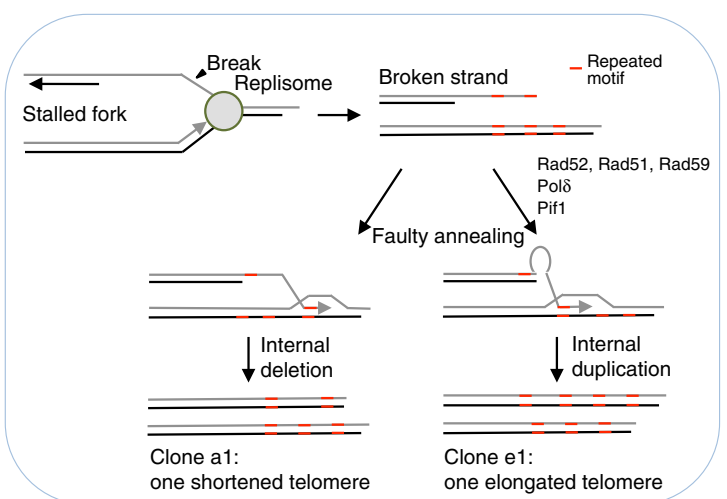

Fig. 5 Analysis of telomeres by DNA sequencing. a Telomeres VI-R were amplified and sequenced from clonal populations of est $2 \Delta$ and est $2 \Delta$ nup1-LexA cells using a specific primer after about 65 population doublings from the initial spores (Day 5) and align with the reference sequence obtained at Day 1 (see Supplementary Fig. 4a for the senescence profiles). Each bar represents an individual telomere. Bars are sorted by the length of the centromereproximal sequence that is identical to the reference sequence (black). The red bars show the length of the distal rearranged sequence that cannot be continuously aligned with the reference. $\mathbf{b}$ Examples of rearrangements. Upper panel, deletion: TeIVI-R from the clone a1 aligns with the reference provided that a 8-bp-long gap is introduced after the 17th nucleotide. Note the repeated motif (in red and blue) at and after the gap. Lower panel, insertion: The 55 first nucleotides of the clone e1 align perfectly with the reference. The last 60 nucleotides (56-86 are shown) cannot be aligned with the reference except if starting at position 29 of the reference instead of 56. This suggests that part of the sequence has been duplicated. Note the presence of the motif (in blue) at the end of the conserved sequence that is repeated just upstream the point of misalignment (in red). c Model of non-conservative HR-dependent repair between sister chromatids. The sequence of the telomeres contains motifs that are repeated several times. Thus a $3^{\prime}$ terminal end with such a motif can anneal at different positions in the sister chromatid to initiate repair leading to internal deletion (left part) or duplication (right part).

SIZ1 and SIZ2 did not alter the attenuated senescence profile conferred by the expression of Nup1-LexA (Supplementary Fig. 6b) indicating that SUMOylation is not a major actor of the repair pathway, that is at play in nup1-LexA cells.

Truncation of the Nup1 C-terminal phenocopies the nup1LexA. Altered telomere recombination in the presence of Nup1LexA might stem from either steric interference instigated by the C-terminal fusion or from the presence of an ectopic DNA binding domain at the pore. To distinguish between these two possibilities, we tested several alternative C-terminal Nup1 fusions. Neither a 13-myc (Supplementary Fig. 7a) nor a CFP tag (Supplementary Fig. 7b) attenuated replicative senescence. In contrast, fusion of the lac repressor LacI to the C-terminal end of Nup1 conferred a phenotype similar to nup1-LexA in the absence of telomerase, i.e., attenuated senescence (Supplementary Fig. 7c) and impaired survivor formation (Supplementary Fig. 7d).

The apparent dependency of the effect on the presence of a DNA binding moiety (LexA or LacI) initially suggested that attenuation of senescence required the DNA-binding activity at 
a

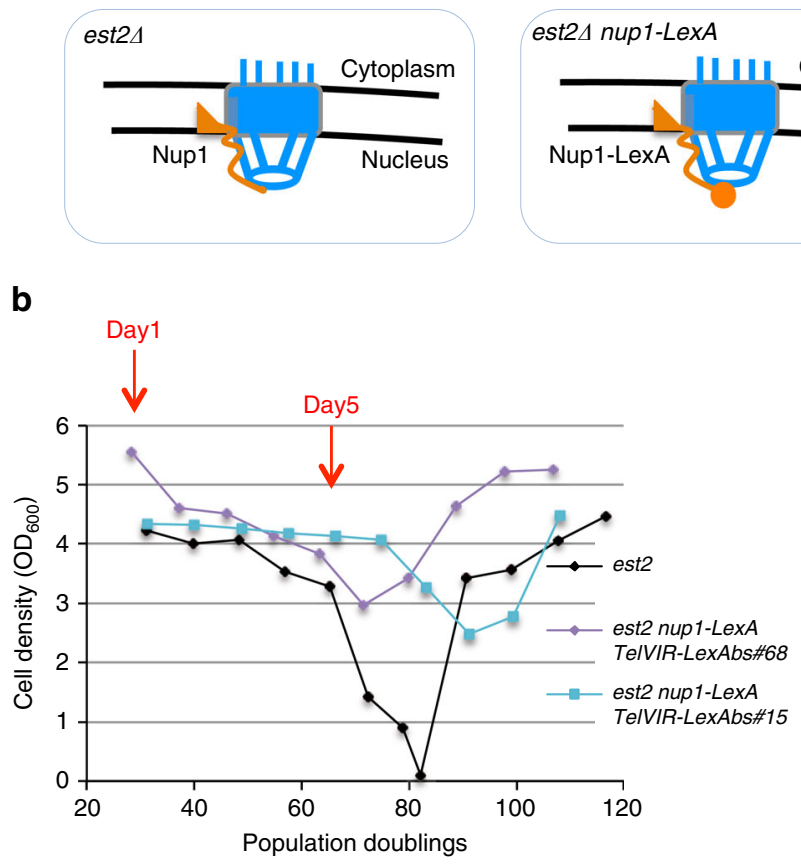

C

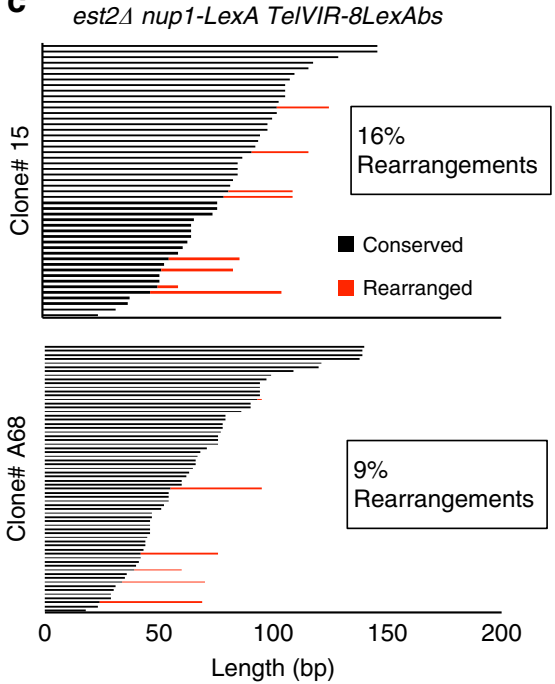

Fig. 6 Tethering of TelVI-R to Nup1-LexA prevents SCR. a Schematic of the three est2 $\Delta$ strains expressing (right panels) or not (left panel) nup1-LexA and bearing either wild-type TeIVI-R or modified TELVI-R with eight LexA DNA-binding sites inserted $1.2 \mathrm{~kb}$ away from the TG $\mathrm{TG}_{1-3}$ repeats $^{35}$. $\mathbf{b}$ Senescence profiles of the two est2 $\Delta$ nup1-LexA TelVI-R-8lexAbs clones (\#15 and \#A68) used for sequencing. The est2 $\Delta$ profile is from Supplementary Fig. 4 and is shown for comparison. D1 and D5 are highlighted in red. c TelVI-R sequence analysis of the clones est2 $\triangle$ nup1-LexA TelVI-R-8LexAbs \#15 and \#A68 at Day 5 (see Supplementary Fig. 5 for the statistics of comparison with WT TelVI-R).

the NPC. We therefore made the assumption that non-specific DNA binding by LexA could interfere with telomere anchoring. We tested whether the overexpression of LexA from a multicopy vector could affect the continuous growth of est2A nup1-LexA cells. As shown in Supplementary Fig. 7e, overexpression of LexA did not affect the behavior of nup1-LexA cells in the absence of telomerase. This result prompt us to test whether the addition of the LexA DNA binding domain to Nup1 impairs a specific Nup1 function required to anchor stalled replication forks to the NPC.

We therefore tested whether loss of function mutants of Nup1 could phenocopy the nup1-lexA phenotypes. Expression of Nup1 $\triangle$ FxFG in which most FG repeats have been removed ${ }^{54}$ neither attenuated senescence (Fig. 7a) nor prevented survivor formation (Fig. 7b). In contrast, we found that expression of Nup1 $\Delta 1040-1076$ (thereafter termed Nup1 $\Delta \mathrm{Ct}$ ) in which the last 36 residues at the $\mathrm{C}$-terminal end have been removed ${ }^{55}$, attenuated senescence in the absence of telomerase to an extent similar to that observed when Nup1-LexA is expressed (Fig. 7c). Accordingly, telomere length analysis showed that, as expected, telomeres shortened as a function of the PDs in the absence of telomerase but unlike est $2 \Delta$ with wild-type NUP1, the est $2 \Delta$ nup $1 \Delta C t$ clones maintained short terminal $\mathrm{TG}_{1-3}$ repeats much longer before displaying some $\mathrm{Y}^{\prime}$ amplification at late time points. The similarity with the phenotype of nup1-LexA prompted us to test whether Nup1 $\Delta$ Ct also affected the relocalization of replicative damages to the NPCs. Figure 7e, f shows that Nup1 $\Delta$ Ct impairs the peripheral enrichment of both the Cdc13-YFP/Rfa1-CFP foci and expanded CAG trinucleotide repeats in est $2 \Delta$ cells.

Based on these results, we concluded that a loss of function of the Nup $1 \Delta \mathrm{Ct}$ protein confers the same phenotypes as those observed in the nup1-LexA strain. We inferred that the LexA fusion somehow impairs a Nup1 anchoring function associated with its C-terminal domain.

\section{Discussion}

Studies over the last years revealed a prominent role of DNA damage relocalization to the nuclear pore in the repair of DNA lesions as diverse as irreparable DSBs, eroded telomeres, collapsed replication forks and replication forks stalled at DNA secondary structures $29,34,37,56$. Although several actors of this pathway have been identified, the functional consequences of the relocalization of damage to the NPC remained difficult to address due to the pleiotropic effects of inactivating pore components ${ }^{42}$. Here we show that disturbing the function of the basket protein Nup1 either by truncation of its C-terminal end or through a fusion with the bacterial DNA-binding protein LexA impairs the localization at the nuclear periphery of replication forks stalled at either expanded CAG tracts or telomeres.

We found that expression of nup1-LexA in cells deprived of telomerase activity profoundly altered the way their telomeres were processed. We demonstrated that interference with the Nup1 C-terminus unleashes a telomere maintenance mechanism that preserves a minimal telomere length compatible with proliferation. This iterative maintenance of $\mathrm{TG}_{1-3}$ repeats by SCR prevents the production of very short and dysfunctional telomeres and hence permanent activation of the checkpoint and replicative senescence. It is not processive enough, however, to produce long telomeres as observed in type II survivors. Remarkably, Nup1-LexA expression in telomerase-negative cells renders cell growth insensitive to RAD5 deletion, which normally accelerates senescence (Supplementary Fig. 4 and ref. ${ }^{47}$ ). Thus, the repair of telomeres that operates specifically in est $2 \Delta$ 
a

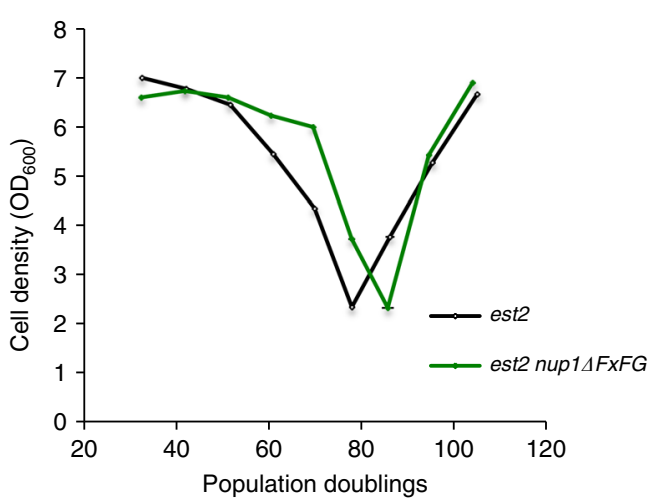

c

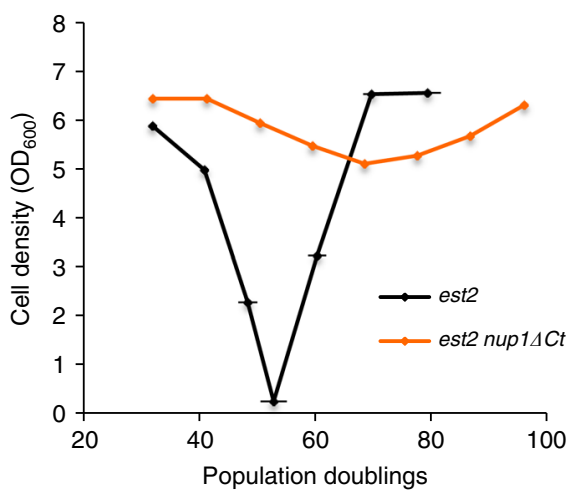

e

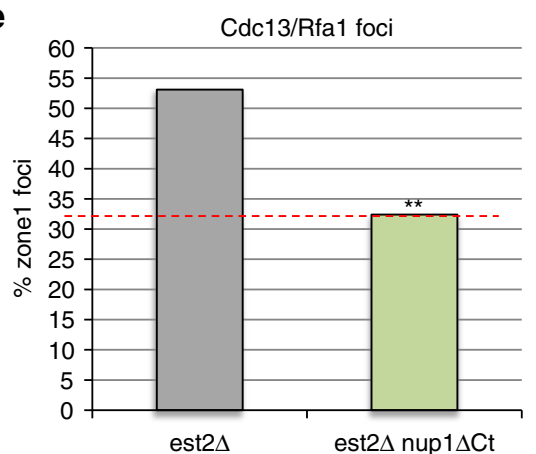

b

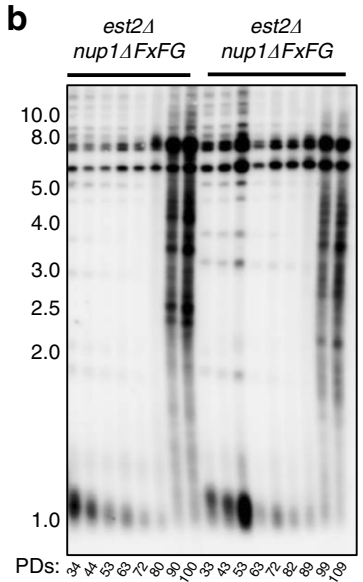

d

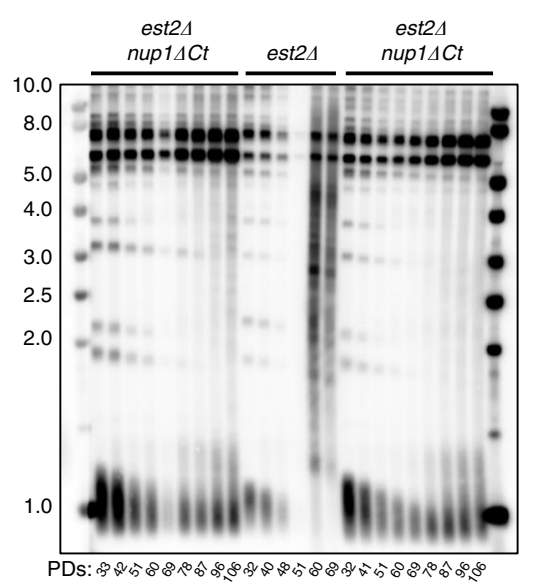

f

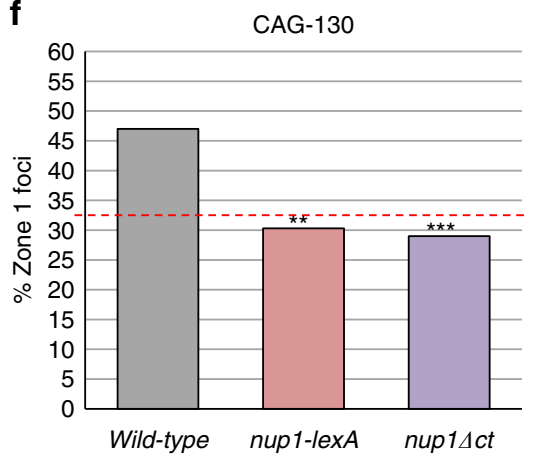

Fig. 7 nup1 $\Delta$ Ct phenocopies the phenotypes of nup1-LexA. a Mean senescence profiles of est2 $\Delta(n=5)$ and est2 $\Delta$ nup1 $\Delta F x F G(n=5)$ clones. Error bars are $\mathrm{SD}$. $\mathbf{b}$ Telomere length analysis of two representative est $2 \Delta$ nup $1 \Delta F x F G$ clones. Southern blot of Xhol-digested DNA prepared from samples of senescing cells was revealed with a $\mathrm{TG}_{1-3}$ probe. c Mean senescence profiles of est $2 \Delta(n=4)$ and est $2 \Delta$ nup $1 \Delta \mathrm{Ct}(n=8)$ clones. Error bars are SD. $\mathbf{d}$ Telomere length analysis of representative clones of the indicated genotypes. $\mathbf{e}$ The localization of the Cdc13-YFP/Rfa1-CFP foci in the three zones of equal area of the nucleus marked by Nic96-RFP was scored in late $\mathrm{S}$ and G2/M est2 $\Delta$ and est2 $\Delta$ nup $1 \Delta \mathrm{Ct}$ cells as described in Fig. 2 . The percentages of zone 1 foci are from three independently isolated est2 $\Delta$ nup $1 \Delta C t$ ( $n=139$ cells) clones. WT cells are from Fig. 3. Statistical differences were determined by a Fisher's exact test $\left.{ }^{* \star} p=0.0019\right)$. f Percent of zone 1 foci for CAG-130 S-phase in nup1 $\Delta C t$ cells $(n=143)$. Statistical differences were determined by a Fisher's exact test $\left({ }^{\star \star *} p=0,0006\right)$. WT and nup1-LexA cells are from Fig. 3. The repartition of the Rfa1/Cdc13 and CAG-130 foci between the three zones of the nucleus is shown in the Supplementary Fig. 8.

nup1-LexA cells seems to be independent of the error-free Rad5dependent branch of the DDT pathway ${ }^{57}$. Instead, our data suggest that in this context, telomeres can be repaired by lowfidelity HR using the sister chromatid as a template. We emphasize that the HR pathway used in this process differs from the pathways leading to survivor formation. First it does not generate long telomeres as observed in type II survivors ${ }^{25}$. Second, it is detected soon after inactivation of the telomerase in contrast to type I and II recombination events that occur at short telomeres during crisis. Finally, the HR at play in est2 2 nup1LexA cells uses sister chromatid as a template instead of another telomere.

We propose that during replicative senescence, NPC relocalization of the forks stalled at telomeres facilitates their repair by a conservative mechanism (Fig. 8, left panel). This pathway might involve the Rad5-dependent branch of the DDT pathway to 

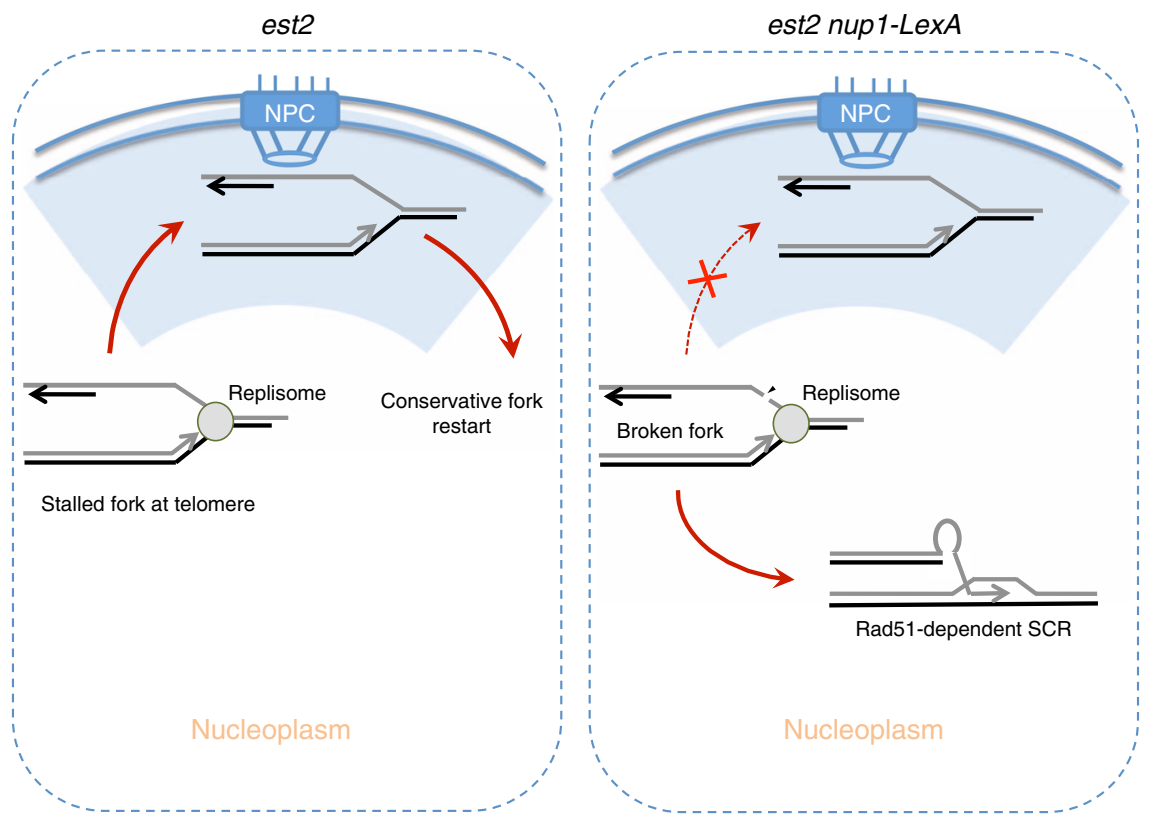

Fig. 8 Model for the role of the NPC in the repair of fork stalled at telomeres. Replication forks stalled at telomeres relocalize to the NPC and are mainly repaired by a conservative pathway that allows replication to resume (left). When this pathway fails, the stalled forks engage in error-prone Rad51dependent SCR that maintain a telomere length compatible with continuous proliferation.

facilitate fork restart since the deletion of RAD5 greatly accelerates senescence ${ }^{47}$. When relocalization to NPCs is impaired, an unrepaired stalled fork might be further processed and possibly break, allowing a default repair through SCR (Fig. 8, right panel). The structure of the fork that localizes to the NPC is unclear, raising the question of the difference between telomeric stalled forks and eroded telomeres, their recognition, and intranuclear partitioning.

Unexpectedly, preventing relocalization of damaged telomeres benefits the cells proliferating in the absence of telomerase, essentially abolishing replicative senescence. It may seem counterintuitive that cells developed a mechanism that actually increases the rate of senescence but yeast cells have evolved for best fitness in the presence of telomerase and do not experience any selective pressure for improved growth in the absence of telomerase. We showed that tethering of stalled fork to the pore favors conservative (error free) fork restart. Selection pressure most probably was on this aspect that is critical for genome stability in telomerase-positive cells. In this respect, the effect of the Nup1-LexA fusion is not limited to influencing the mechanism of telomere maintenance in telomerase-negative cells since it also increases the instability of CAG-triplet repeats (Fig. 3c). It is well established that long CAG tracts stall replication forks due to formation of hairpin structures $50,58,59$. Expanded CAG tracts transiently associate with the nuclear pore during late $S$ phase in budding yeast, which prevents HRdependent instability and may promote replication fork restart ${ }^{37}$. Previous work indicates that inactivation of the Nup84 protein prevents relocalization of triplet repeats to the NPCs and increases CAG repeat instability, especially Rad52-dependent contractions. Strikingly, we show here that Rad52-dependent CAG repeat contractions are also increased in the nup1-LexA strain. Therefore, the role of the NPC in restricting error-prone recombination between repeated sequences appears to be acting at two different types of fork-stalling sequences, telomeres and CAG/CTG repeats. This mechanism may be a universal way to control recombination during repair of stalled or collapsed forks to protect genome integrity.
The question remained why fusion of LexA to Nup1 affects the relocalization of the stalled forks to the NPCs? We discovered that truncation of the $36 \mathrm{C}$-terminal residues of Nup1 impacts the senescence profile and the localization of stalled forks at telomeres and at CAG triplet repeats at a similar extent as the Cterminal LexA fusion. Remarkably, the function of Nup1 in the processing of stalled replication forks appears to be specific since inactivation of the other basket proteins, i.e., Nup60 or Mlp1 and Mlp2, does not attenuate telomere-induced senescence (Supplementary Fig. 9a and ref. ${ }^{35}$ ). It is possible that the C-terminal domain of Nup1 may interact directly with one of the proteins bound to the stalled forks. Currently, the only function attributed to the unstructured C-terminal domain of Nup1 is that it contributes to the high-affinity interaction of Nup1 with the Kap60Kap95-cargo complexes, although the way it does so remains elusive 55,60 . It has been proposed that the strong interaction of Nup1 with Kap95-Kap60 might help to guide the cargo to the correct site ${ }^{60}$. Although the nup1-LexA and nup1 $\Delta C t$ alleles differ by their genetic interaction with the deletion of nup60 4 (Supplementary Fig. 9b, c), suggesting that interactions with the karyopherins is less affected by the LexA fusion than by the Cterminal deletion, one exciting possibility is that Nup1 regulates a secondary role of the karyopherins in escorting a cargo to the site of replication stress. Such docking functions, independent of the transport function per se, have already been attributed to karyopherins in tethering Ulp1 to the $\mathrm{NPC}^{61}$ or in stabilizing the Dam 1 complex until it associates with spindle microtubules ${ }^{62}$. In this scheme, Nup1-Kap-cargo interaction would contribute to the loading of the cargo onto the stalled replication fork either directly at the NPC or in the nucleoplasm before relocalization to the nuclear pore, whereby shaping the fork for conservative restart. Understanding the way Nup1-LexA and Nup1 $\Delta$ Ct interfere with the stalled forks attempting to interact with the NPCs will require further investigation.

Numerous actors participate in the response to DNA replication perturbations. Together, our data reinforce the pivotal role of the NPC to favor the most conservative pathway to rescue stalled replication forks. The need to preserve the fork against 
recombination is probably more critical at repeated sequences where faulty annealing can lead to instability. It remains unclear whether the role of the NPC upon replication stress is preserved in metazoans but intranuclear positioning also participates in DNA repair pathway choice in human cells ${ }^{63}$. Repair pathway control might be fundamental for human health considering the role of trinucleotide repeat expansion in several diseases ${ }^{64}$. This is also true at telomeres since the iterative recombination between sister chromatids described here can thwart senescence, a potent anti-tumor mechanism in mammalian cells. Interestingly, a recent survey of 6835 cancers showed that $22 \%$ of tumors neither express telomerase at detectable level nor harbored characteristics of $\mathrm{ALT}^{65}$. In line with this unexpected observation, cases of metastatic melanoma cells $^{66}$ and high-risk neuroblastoma ${ }^{67}$ have been reported that lacked significant canonical telomere lengthening mechanisms. We propose that unleashed SCR might constitute the first step in bypassing the proliferation barrier induced by unprotected telomeres during carcinogenesis, before full telomere stabilization takes place via either telomerase reactivation or ALT.

\section{Methods}

Yeast strains. Strains used in this study are described in Supplementary Table 1. Strains were constructed and analyzed by standard genetic methods.

Senescence assays. Liquid senescence assays were performed starting with the haploid spore products of diploids that were heterozygous for EST2 (EST2/est2A) and for the gene(s) of interest. To ensure homogeneous telomere length before sporulation, each diploid has been propagated for at least 50 PDs on YPD plates. After 2-3 days of growth at $30^{\circ} \mathrm{C}$, the entire spore colonies were transferred to $2 \mathrm{ml}$ liquid YPD to estimate the number of PDs and the suspension immediately diluted to $10^{5}$ cells per milliliter. Cells were serially passaged in $15 \mathrm{ml}$ of liquid YPD medium at $10^{5}$ cells per milliliter at 24 -h intervals. Replicative senescence curves shown in this study correspond to the average of several independent spores with identical genotype. Senescence assays on solid medium were initiated as described above, but the cells were propagated by consecutive restreaking on solid YPD plates followed by outgrowth for 2 days at $30^{\circ} \mathrm{C}$. The process was repeated until the appearance of survivors.

Telomere sequence analysis. The telomere TELVI- $R$ was amplified by PCR using the AccuPrime ${ }^{\text {mot }}$ GC-Rich DNA Polymerase (InVitrogen) and the TELVI-R specific primer 5'-CGTGTGCGTACGCCATATCAATATG-3' and cloned into TA-cloning vector (InVitrogen ${ }^{24}$. Sequencing was perfomed by GATC BIOTECH. Telomere sequences were aligned using EMBOSS needle website. Conserved sequences were defined when perfectly matching to the consensus and upon single point mutation or 1-2-bp deletion/insertions. The point of divergence was defined when the sequence could not be aligned with the reference sequence.

Telomere southern blot analysis. A total of $25 \mu \mathrm{g}$ of genomic DNA was digested with $\mathrm{XhoI}$ overnight at $37^{\circ} \mathrm{C}$. Digested DNA was resolved in $0.9 \%$ agarose gel and transferred onto an XL nylon membrane. The DNA was hybridized with a radiolabeled DNA fragment composed of $\mathrm{TG}_{1-3}$ repeats.

Fluorescence microscopy. Fluorescence in situ hybridization (FISH) was performed as described ${ }^{68}$. Cells grown in YPD at $30^{\circ} \mathrm{C}$ were fixed with $4 \%$ final paraformaldehyde to the media. The percentage of cells with poly(A)+-RNA accumulation was calculated from at least 250 cells per condition. Protein import and export from the nucleus were monitored using plasmids encoding GFP-NLS or GFP-NLS-NES ${ }^{69}$, respectively. Observations of exponentially growing cells were performed using a Nikon Eclipse Ti microscope with a 100× objective. Cell images were captured with a Neo sCMOS Camera (Andor). For each field of view, 11 stacks were acquired at $0.3-\mu \mathrm{m}$ intervals along the $Z$-axis. Because the GFP-NLS tends to form aggregates in the nucleus, total fluorescence intensity of each cell was measured as well as the fluorescence in the nucleus using ImageJ software. The nuclear/cytoplasmic ratio $(N / C)$ was calculated on maximum intensity projection for each cell as ((nuclear fluorescence-background)/nuclear area) / ((total fluorescence-background) - (nuclear fluorescence-background) / (cell area-nuclear area)). For GFP-NLS-NES, the nucleocytoplasmic ratio was measured at the focal plane using the ROI plugin of ImageJ. Mean fluorescence intensity within constant square regions placed in the cytoplasm, the nucleus marked with NIC96-RFP and in the intercellular background was measured. $\mathrm{N} / \mathrm{C}$ ratios are

(Nucleus-background) / (Cytoplasm-background).

Cdc13-YFP/Rfa1-CFP zoning assay: Spore colonies from a heterozygous est2 $\triangle$ nup1-LexA CDC13-YFP RFA1-CFP NIC96-RFP diploid were streaked once on YPD plates supplemented with adenine ( $6 \mathrm{mg}$ per milliliter) and grown for $48 \mathrm{~h}$ (about 35
PDs from the original spore). Cells were then grown overnight at low density on YPD plates supplemented with adenine before imaging. Observations were performed using a Nikon Eclipse Ti microscope with a 100× objective and a binning $2 \times$. Cell images were captured with a Neo sCMOS Camera (Andor) as Z-stack of 21 images with a step interval of $0.2 \mu \mathrm{m}$. RfA1-CFP and Cdc13-YFP co-localization was assessed at each Z-plane. The zoning assay was then performed using median filtered Rfa1CFP and Nic96-RFP stacks at the Z-plane of Cdc13/Rfa1 co-localization using the point-picker plugin in ImageJ software ${ }^{41}$. Late S-phase cells were defined as cells with a medium bud and a single nucleus in the mother cells. G2/M cells were defined as cells with the nucleus at or spanning the bud neck. As the nuclei are often bi-lobed between the mother and the daughter at the G2/M transition, only the lobe containing the foci was considered for measurement.

CAG repeat zoning assay: Colonies were checked for presence of 130 CAG repeats by PCR with primers flanking the repeat. Cells from colonies with the correct repeat length were grown to $\sim 5 \times 10^{6}$ cells per milliliter in YC media, and a portion was rechecked to confirm the tract length had been maintained. Cells were fixed with $4 \%$ paraformaldehyde. Z-stack images were taken using a Zeiss AX10 fluorescent microscope under 100× magnification. Step interval size was $0.15 \mu \mathrm{m}$ and $\sim 30$ images were taken per stack. Exposure time was DIC: $100 \mathrm{~ms}$; GFP: $500 \mathrm{~ms}$. Images were deconvolved, and three-zoning criteria was used to evaluate the location of the GFP foci for S-phase cells with the ImageJ point picker program ${ }^{41}$. S-phase cells were determined by yeast morphology as described in ref. ${ }^{70}$. Data for each assay were obtained from two independent nup 1-LexA strains, and three independent experiments were performed (two for strain \#4470 and one for strain \#4469).

Triplet repeat stability and fragility assays. The CAG tract was amplified from yeast colonies using primers spanning the repeats (P1 and P2 in Supplementary Fig. 3a) as described in Sundararajan et al. ${ }^{71}$ to confirm correct tract length. Colonies were grown in YC-Leu liquid media for six to seven cell divisions to allow expansion, contraction or breakage, and were plated on FOA-Leu and YC-Leu plates. For the instability assay, CAG repeat length in at least 100 daughter colonies from at least four different parent colonies on the YC-Leu plates was assessed by PCR amplification and sizing of the amplicons on an AATI fragment analyzer system. Fragments 3 or more repeats longer or shorter than the starting tract length were counted as expansions or contractions, respectively. Data for each assay were obtained from two independent nup1-LexA strains. To assay fragility, colonies growing on FOA-Leu and YC-Leu were counted, and a rate of mutation was calculated using the method of the median ${ }^{72}$. At least three independent 10 colony fluctuation assays were performed per strain. A subset of $\mathrm{FOA}^{\mathrm{R}}$ colonies was checked for end loss (primers P3 and P4 in Supplementary Fig. 3a) and this occurred at a similar frequency in wild-type and nup1-LexA mutants.

Reporting summary. Further information on research design is available in the Nature Research Reporting Summary linked to this article.

\section{Data availability}

The data that support the findings of the current study are available from the corresponding authors on reasonable request. The individual senescence curves underlying Figs. 1b-e, 7a, c and Supplementary Figs. 5b, 6a, b, 7a-c, e, and 8 as well as the uncropped and unprocessed scans of the Southern blots of the main figures are provided as a Source Data file.

Received: 27 February 2019; Accepted: 10 December 2019; Published online: 09 January 2020

\section{References}

1. Larrivee, M., LeBel, C. \& Wellinger, R. J. The generation of proper constitutive G-tails on yeast telomeres is dependent on the MRX complex. Genes Dev. 18, 1391-1396 (2004)

2. Stewart, J. A., Chaiken, M. F., Wang, F. \& Price, C. M. Maintaining the end: roles of telomere proteins in end-protection, telomere replication and length regulation. Mutat. Res. 730, 12-19 (2012).

3. Pardo, B. \& Marcand, S. Rap1 prevents telomere fusions by nonhomologous end joining. EMBO J. 24, 3117-3127 (2005)

4. Martina, M. et al. A balance between Tel1 and Rif2 activities regulates nucleolytic processing and elongation at telomeres. Mol. Cell. Biol. 32, 1604-1617 (2012).

5. Bonetti, D. et al. Shelterin-like proteins and Yku inhibit nucleolytic processing of Saccharomyces cerevisiae telomeres. PLoS Genet. 6, e1000966 (2010).

6. Graf, M. et al. Telomere length determines TERRA and R-Loop regulation through the cell cycle. Cell 170, 72-85.e14 (2017).

7. Wellinger, R. J. \& Zakian, V. A. Everything you ever wanted to know about Saccharomyces cerevisiae telomeres: beginning to end. Genetics 191, 1073-1105 (2012).

8. Paeschke, K., McDonald, K. R. \& Zakian, V. A. Telomeres: structures in need of unwinding. FEBS Lett. 584, 3760-3772 (2010). 
9. Higa, M., Fujita, M. \& Yoshida, K. DNA replication origins and fork progression at mammalian telomeres. Genes (Basel) 8, E112 (2017).

10. Lingner, J., Cooper, J. P. \& Cech, T. R. Telomerase and DNA end replication: no longer a lagging strand problem? Science 269, 1533-1534 (1995).

11. Hector, R. E. et al. Meclp associates with functionally compromised telomeres. Chromosoma 121, 277-290 (2012).

12. A.S., I. \& Greider, C. W. Short telomeres induce a DNA damage response in Saccharomyces cerevisiae. Mol. Biol. Cell 14, 987-1001 (2003).

13. Dehe, P. M., Rog, O., Ferreira, M. G., Greenwood, J. \& Cooper, J. P. Taz1 enforces cell-cycle regulation of telomere synthesis. Mol. Cell 46, 797-808 (2012).

14. Simon, M. N., Churikov, D. \& Geli, V. Replication stress as a source of telomere recombination during replicative senescence in Saccharomyces cerevisiae. FEMS Yeast Res. 16 (2016).

15. Maestroni, L., Matmati, S. \& Coulon, S. Solving the telomere replication problem. Genes (Basel) 8, 55 (2017).

16. Lundblad, V. \& Blackburn, E. H. An alternative pathway for yeast telomere maintenance rescues est1- senescence. Cell 73, 347-360 (1993).

17. Xie, Z. et al. Early telomerase inactivation accelerates aging independently of telomere length. Cell 160, 928-939 (2015).

18. $\mathrm{Xu}, \mathrm{Z}$. et al. Two routes to senescence revealed by real-time analysis of telomerase-negative single lineages. Nat. Commun. 6, 7680 (2015).

19. McEachern, M. J. \& Haber, J. E. Break-induced replication and recombinational telomere elongation in yeast. Annu. Rev. Biochem. 75, 111-135 (2006).

20. $\mathrm{Hu}, \mathrm{Y}$. et al. Telomerase-null survivor screening identifies novel telomere recombination regulators. PLoS Genet. 9, e1003208 (2013).

21. Teng, S. C. \& Zakian, V. A. Telomere-telomere recombination is an efficient bypass pathway for telomere maintenance in Saccharomyces cerevisiae. Mol. Cell. Biol. 19, 8083-8093 (1999).

22. Lee, J. Y., Kozak, M., Martin, J. D., Pennock, E. \& Johnson, F. B. Evidence that a RecQ helicase slows senescence by resolving recombining telomeres. PLoS Biol. 5, el60 (2007).

23. Le, S., Moore, J. K., Haber, J. E. \& Greider, C. W. RAD50 and RAD51 define two pathways that collaborate to maintain telomeres in the absence of telomerase. Genetics 152, 143-152 (1999).

24. Hardy, J., Churikov, D., Geli, V. \& Simon, M. N. Sgs1 and Sae2 promote telomere replication by limiting accumulation of ssDNA. Nat. Commun. $\mathbf{5}$, 5004 (2014).

25. Chen, Q., Ijpma, A. \& Greider, C. W. Two survivor pathways that allow growth in the absence of telomerase are generated by distinct telomere recombination events. Mol. Cell. Biol. 21, 1819-1827 (2001).

26. Kalousi, A. \& Soutoglou, E. Nuclear compartmentalization of DNA repair. Curr. Opin. Genet. Dev. 37, 148-157 (2016).

27. Geli, V. \& Lisby, M. Recombinational DNA repair is regulated by compartmentalization of DNA lesions at the nuclear pore complex. Bioessays 37, 1287-1292 (2015).

28. Kalocsay, M., Hiller, N. J. \& Jentsch, S. Chromosome-wide Rad51 spreading and SUMO-H2A.Z-dependent chromosome fixation in response to a persistent DNA double-strand break. Mol. Cell 33, 335-343 (2009).

29. Nagai, S. et al. Functional targeting of DNA damage to a nuclear poreassociated SUMO-dependent ubiquitin ligase. Science 322, 597-602 (2008).

30. Hayama, R., Rout, M. P. \& Fernandez-Martinez, J. The nuclear pore complex core scaffold and permeability barrier: variations of a common theme. Curr. Opin. Cell Biol. 46, 110-118 (2017).

31. Bennett, C. B. et al. Genes required for ionizing radiation resistance in yeast. Nat. Genet. 29, 426-434 (2001).

32. Palancade, B. et al. Nucleoporins prevent DNA damage accumulation by modulating Ulp1-dependent sumoylation processes. Mol. Biol. Cell 18, 2912-2923 (2007).

33. Loeillet, S. et al. Genetic network interactions among replication, repair and nuclear pore deficiencies in yeast. DNA Repair 4, 459-468 (2005).

34. Khadaroo, B. et al. The DNA damage response at eroded telomeres and tethering to the nuclear pore complex. Nat. Cell Biol. 11, 980-987 (2009).

35. Churikov, D. et al. SUMO-dependent relocalization of eroded telomeres to nuclear pore complexes controls telomere recombination. Cell Rep. 15, 1242-1253 (2016).

36. Horigome, C. \& Gasser, S. M. SUMO wrestles breaks to the nuclear ring's edge. Cell Cycle 15, 3011-3013 (2016).

37. Su, X. A., Dion, V., Gasser, S. M. \& Freudenreich, C. H. Regulation of recombination at yeast nuclear pores controls repair and triplet repeat stability. Genes Dev. 29, 1006-1017 (2015).

38. Harper, N. C., Al-Greene, N. T., Basrai, M. A. \& Belanger, K. D. Mutations affecting spindle pole body and mitotic exit network function are synthetically lethal with a deletion of the nucleoporin NUP1 in S. cerevisiae. Curr. Genet. 53, 95-105 (2008).

39. Krogh, B. O. \& Symington, L. S. Recombination proteins in yeast. Annu. Rev. Genet. 38, 233-271 (2004).
40. Lisby, M. \& Geli, V. DNA damage response to eroded telomeres. Cell Cycle 8, 3617-3618 (2009)

41. Meister, P., Gehlen, L. R., Varela, E., Kalck, V. \& Gasser, S. M. Visualizing yeast chromosomes and nuclear architecture. Methods Enzymol. 470, 535-567 (2010).

42. Freudenreich, C. H. \& Su, X. A. Relocalization of DNA lesions to the nuclear pore complex. FEMS Yeast Res. 16, fow095 (2016).

43. Dion, V., Kalck, V., Horigome, C., Towbin, B. D. \& Gasser, S. M. Increased mobility of double-strand breaks requires Mec1, Rad9 and the homologous recombination machinery. Nat. Cell Biol. 14, 502-509 (2012).

44. Forstemann, K. \& Lingner, J. Molecular basis for telomere repeat divergence in budding yeast. Mol. Cell. Biol. 21, 7277-7286 (2001).

45. Claussin, C. \& Chang, M. Multiple Rad52-mediated homology-directed repair mechanisms are required to prevent telomere attrition-induced senescence in Saccharomyces cerevisiae. PLoS Genet. 12, el006176 (2016).

46. Anand, R., Beach, A., Li, K. \& Haber, J. Rad51-mediated double-strand break repair and mismatch correction of divergent substrates. Nature 544, 377-380 (2017).

47. Fallet, E. et al. Length-dependent processing of telomeres in the absence of telomerase. Nucleic Acids Res. 42, 3648-3665 (2014).

48. Azam, M. et al. Evidence that the S.cerevisiae Sgs1 protein facilitates recombinational repair of telomeres during senescence. Nucleic Acids Res. 34, 506-516 (2006).

49. Lambert, S. et al. Homologous recombination restarts blocked replication forks at the expense of genome rearrangements by template exchange. Mol. Cell 39, 346-359 (2010).

50. Nguyen, J. H. G. et al. Differential requirement of Srs 2 helicase and Rad51 displacement activities in replication of hairpin-forming CAG/CTG repeats. Nucleic Acids Res. 45, 4519-4531 (2017).

51. Segurado, M. \& Diffley, J. F. Separate roles for the DNA damage checkpoint protein kinases in stabilizing DNA replication forks. Genes Dev. 22, 1816-1827 (2008)

52. Szyjka, S. J. et al. Rad53 regulates replication fork restart after DNA damage in Saccharomyces cerevisiae. Genes Dev. 22, 1906-1920 (2008).

53. Bermejo, R. et al. The replication checkpoint protects fork stability by releasing transcribed genes from nuclear pores. Cell 146, 233-246 (2011).

54. Strawn, L. A., Shen, T., Shulga, N., Goldfarb, D. S. \& Wente, S. R. Minimal nuclear pore complexes define FG repeat domains essential for transport. Nat. Cell Biol. 6, 197-206 (2004).

55. Pyhtila, B. \& Rexach, M. A gradient of affinity for the karyopherin Kap95p along the yeast nuclear pore complex. J. Biol. Chem. 278, 42699-42709 (2003).

56. Oza, P., Jaspersen, S. L., Miele, A., Dekker, J. \& Peterson, C. L. Mechanisms that regulate localization of a DNA double-strand break to the nuclear periphery. Genes Dev. 23, 912-927 (2009).

57. Branzei, D. \& Szakal, B. DNA damage tolerance by recombination: molecular pathways and DNA structures. DNA Repair (Amst.) 44, 68-75 (2016).

58. Samadashwily, G. M., Raca, G. \& Mirkin, S. M. Trinucleotide repeats affect DNA replication in vivo. Nat. Genet. 17, 298-304 (1997).

59. Pelletier, R., Krasilnikova, M. M., Samadashwily, G. M., Lahue, R. \& Mirkin, S M. Replication and expansion of trinucleotide repeats in yeast. Mol. Cell. Biol. 23, 1349-1357 (2003).

60. Liu, S. M. \& Stewart, M. Structural basis for the high-affinity binding of nucleoporin Nuplp to the Saccharomyces cerevisiae importin-beta homologue, Kap95p. J. Mol. Biol. 349, 515-525 (2005).

61. Panse, V. G., Kuster, B., Gerstberger, T. \& Hurt, E. Unconventional tethering of Ulp1 to the transport channel of the nuclear pore complex by karyopherins. Nat. Cell Biol. 5, 21-27 (2003).

62. Cairo, L. V. \& Wozniak, R. W. The nuclear transport factor Kap121 is required for stability of the Daml complex and mitotic kinetochore biorientation. Cell Rep. 14, 2440-2450 (2016).

63. Lemaitre, C. et al. Nuclear position dictates DNA repair pathway choice. Genes Dev. 28, 2450-2463 (2014).

64. Jones, L., Houlden, H. \& Tabrizi, S. J. DNA repair in the trinucleotide repeat disorders. Lancet Neurol. 16, 88-96 (2017).

65. Barthel, F. P. et al. Systematic analysis of telomere length and somatic alterations in 31 cancer types. Nat. Genet. 49, 349-357 (2017).

66. Dagg, R. A. et al. Extensive proliferation of human cancer cells with evershorter telomeres. Cell Rep. 19, 2544-2556 (2017).

67. Viceconte, $\mathrm{N}$. et al. Highly aggressive metastatic melanoma cells unable to maintain telomere length. Cell Rep. 19, 2529-2543 (2017).

68. Babour, A. et al. The chromatin remodeler ISW1 is a quality control factor that surveys nuclear mRNP biogenesis. Cell 167, 1201-1214.e15 (2016).

69. Nino, C. A. et al. Posttranslational marks control architectural and functional plasticity of the nuclear pore complex basket. J. Cell Biol. 212, 167-180 (2016).

70. Hediger, F., Taddei, A., Neumann, F. R. \& Gasser, S. M. Methods for visualizing chromatin dynamics in living yeast. Methods Enzymol. 375, 345-365 (2004). 
71. Sundararajan, R., Gellon, L., Zunder, R. M. \& Freudenreich, C. H. Doublestrand break repair pathways protect against CAG/CTG repeat expansions, contractions and repeat-mediated chromosomal fragility in Saccharomyces cerevisiae. Genetics 184, 65-77 (2010).

72. Polleys, E. J. \& Freudenreich, C. H. Methods to study repeat fragility and instability in Saccharomyces cerevisiae. Methods Mol. Biol. 1672, 403-419 (2018).

\section{Acknowledgements}

We thank Michael Lisby, Susan Wente and Hannah Klein for generous gifts of strains, Anna Babour for help with FISH experiment and Marion Dubarry, Daniel Isnardon and Davide Normanno for help with microscopy and processing of images. P.A. was supported by the Région Provence-Alpes-Côte d'Azur and the Association pour la Recherche contre le Cancer (ARC). C.M. was supported by L'Institut National Du Cancer (INCA, PLBIO14-012). V.G. is supported by the Ligue Nationale Contre le Cancer (équipe labellisée) and by INCA (PLBIO14-012). C.H.F. funding for this work was from NIH R01 grant GM122880.

\section{Author contributions}

P.A., C.M., D.C. and M.N.S. designed and performed all experiments except the CAG trinucleotide repeat experiments that were designed and performed by J.W. and C.F. D.C. and C.F. discussed the results and contributed to the writing of the manuscript. M.N.S. and V.G. conceived this study and wrote the manuscript.

\section{Competing interests}

The authors declare no competing interests.

\section{Additional information}

Supplementary information is available for this paper at https://doi.org/10.1038/s41467019-13979-5.

Correspondence and requests for materials should be addressed to M.-N.S. or V.G.

Peer review information Nature Communications thanks the anonymous reviewer(s) for their contribution to the peer review of this work. Peer reviewer reports are available.

Reprints and permission information is available at http://www.nature.com/reprints

Publisher's note Springer Nature remains neutral with regard to jurisdictional claims in published maps and institutional affiliations.

(c) (i) Open Access This article is licensed under a Creative Commons Attribution 4.0 International License, which permits use, sharing, adaptation, distribution and reproduction in any medium or format, as long as you give appropriate credit to the original author(s) and the source, provide a link to the Creative Commons license, and indicate if changes were made. The images or other third party material in this article are included in the article's Creative Commons license, unless indicated otherwise in a credit line to the material. If material is not included in the article's Creative Commons license and your intended use is not permitted by statutory regulation or exceeds the permitted use, you will need to obtain permission directly from the copyright holder. To view a copy of this license, visit http://creativecommons.org/ licenses/by/4.0/.

(C) The Author(s) 2020 